Melissa de CAstro Abelha ${ }^{1}$

ROSALY RULLI COSTA ${ }^{2}$

VINICIUS MEDINA LOPES ${ }^{3}$

Rachel COSTA Vinhaes dos Reis ${ }^{4}$

Carla Maria Martins Da Silva ${ }^{5}$

\section{Recanalização tubária: análise dos resultados de 30 anos de tratamento}

\author{
Tubal reanastomosis: analysis of the results of 30 years of treatment
}

Artigos originais

Palavras-chave

Microcirurgia

Anastomose cirúrgica Reversão da esterilizaçção

Taxa de gravidez

Gravidez ectópica

Resultado de tratamento

Keywords

Microsurgery

Anastomosis, surgical

Sterilization reversal

Pregnancy rate

Pregnancy, ectopic

Treatment outcome

\section{Resumo}

OBJETIVO: verificar a freqüência de gestação intra-uterina em pacientes que foram submetidas à recanalização tubária no Hospital Regional da Asa Sul nos últimos 30 anos e avaliar o índice de gestação ectópica destes procedimentos e a influência da idade e do tempo decorrido entre a laqueadura e a recanalização no sucesso terapêutico. MÉTODOS: foram analisados 71 prontuários, tendo sido excluídos os que apresentavam outras alterações que pudessem influenciar no prognóstico de fertilidade do casal e os casos em que não foi possível a cirurgia de recanalização. As variáveis coletadas foram: a ocorrência de gestação intra-uterina, chegando esta a termo ou a abortamento; a ocorrência de gestação ectópica após a cirurgia; a não-concepção após a reversão; a idade das mulheres na data da recanalização e o tempo decorrido entre a laqueadura e a sua reversão. RESULTADOS: verificaram-se um índice de gravidez de $67,6 \%$, sendo $73,2 \%$ para as recanalizações bilaterais e $46,6 \%$ para as unilaterais, e um percentual de gravidez ectópica de 5,6\%. Nas faixas etárias entre 20 e 24 anos, foi observado 33\% de gravidez; entre 25 e 29, 60\%; entre 30 e 34, 69,2\%; entre 35 e 39, 65\%, e entre 40 e 44, 42,9\%. A casuística foi pequena para análise dos grupos de 20 e 24 e 40 e 44 anos. $\bigcirc$ intervalo de tempo entre a laqueadura e a recanalização (ITLR) variou de um a 18 anos. $\bigcirc$ intervalo de tempo entre a ITLR foi dividido em três grupos que apresentaram as taxas de gravidez: de um a seis anos, 59\%; de sete a 12 anos, 66,6\%; de 13 a 18 anos, 57\%. CONCLUSÕES: o índice de gestação foi de $67,6 \%$, sendo $5,6 \%$, ectópicas. Comparando as faixas etárias, não houve influência significativa da idade no sucesso terapêutico entre 25 e 39 anos. Verificou-se que não houve influência do tempo de esterilidade sobre os resultados da reversão.

\section{Abstract}

PURPOSE: to verify the ratio of intra-uterine gestation in patients submitted to recanalization in the Hospital Regional da Asa Sul in the last 30 years and to assess the rate of ectopic gestation of such procedures, the influence of age and time interval between salpingectomy and recanalization in the therapeutic success. METHODS: medical files of 71 patients were analyzed, after exclusion of those presenting other alterations that could influence fertility prognosis, plus the cases when recanalization was impossible. Variables collected were: occurrence of intra-uterine gestation, coming to term or to abortion; occurrence of ectopic pregnancy after salpingectomy; no-conception after reversion, women's age at the recanalization, and time interval between salpingectomy and its reversion. RESULTS: there has been a pregnancy rate of $67.6 \%, 73.2 \%$ for bilateral recanalization and $46.6 \%$ for unilateral, as well as $5.6 \%$ of ectopic pregnancies. Concerning the patients' age group, it was observed a pregnancy rate of $33 \%$, from 20 to $24 ; 60 \%$, from 25 to 29 ; $69.2 \%$, from 30 to $34 ; 65 \%$, from 35 to 39 , and $42.9 \%$, from 40 to 44 years old. The number of cases was small for age the groups 20 to 24 and 40 to 44 years old. The time interval between salpingectomy and recanalization (TISR) has varied from one to 18 years. TISR has been divided in three groups presenting the following pregnancy rates: one to six year interval, 59\%; seven to $12,66.6 \%$; 13 to 18,57\%. CONCLUSIONS: gestation rate has been $67.6 \%, 5.6 \%$ being ectopic. In the comparison of age groups, there has been no significant influence of age on the therapeutic success of patients from 25 to 39 years old. Sterility duration did not influence the reversion results.
Correspondência:

Rosaly Rulli Costa

SHIS Q1 17, Coniunto 4, casa 18 CEP 71645-040 - Brasilia/DF Fone: (61) 3552-3960/(61) 81856506/Fax: (61) 3352-6822 E-mail: rrullicosta@yahoo.com.br

Recebido $10 / 4 / 2008$

\section{Hospital Regional da Asa Sul - HRAS - Brasília (DF), Brasil.}

Residente do Programa de Residência Médica em Reprodução Humana do Hospital Regional da Asa Sul - HRAS - Brasília (DF), Brasil.

${ }^{2}$ Coordenadora do Programa de Residência Médica em Reprodução Humana do Hospital Regional da Asa Sul - HRAS - Brasília (DF), Brasil.

${ }^{3}$ Consultor Científico do Serviço de Reprodução Humana do Hospital Regional da Asa Sul - HRAS - Brasília (DF), Brasil.

${ }^{4}$ Médica do Serviço de Reprodução Humana do Hospital Regional da Asa Sul - HRAS - Brasília (DF), Brasil.

${ }_{5}^{5}$ Preceptora do Programa de Residência Médica em Reprodução Humana do Hospital Regional da Asa Sul - HRAS - Brasília (DF), Brasil. 


\section{Introdução}

A laqueadura tubária é um método eficaz de anticoncepção, levando muitos casais em todo o mundo, a escolherem essa opção para limitar o número de filhos ${ }^{1}$. Aproximadamente 138 milhões de mulheres no mundo, em idade reprodutiva, já foram submetidas à laqueadura das tubas ${ }^{2}$.

O Brasil tem um dos mais altos índices de esterilização feminina no mundo ${ }^{3}$. Dados demográficos nacionais mostram que, entre mulheres entrevistadas na faixa etária de 25 a 29 anos, a prevalência de laqueadura é de 21,1\%, e, de 30 a 34 anos, é de $37,6 \%$. As taxas mais altas foram encontradas entre as mulheres com união estável, sendo as porcentagens nestes casos de $26,9 \%$, para o grupo entre 25 e 29 anos e $42,7 \%$, para o grupo entre 30 e 34 anos $^{4}$.

A legislação brasileira preconiza, através da lei $\mathrm{n}^{\circ} 9.263$ de 12 de janeiro de 1996, como condição para realização da esterilização cirúrgica da mulher, que haja um termo de consentimento livre e esclarecido evidenciando a manifestação da vontade. Neste, é expressa a dificuldade da reversão e a existência de outros métodos reversíveis de contracepção. Porém, entre as mulheres que optam pela laqueadura, muitas se arrependem, especialmente as que se submeteram ao procedimento entre os 20 e $30 \operatorname{anos}^{1,5}$. O índice de arrependimento varia entre 2 e $13 \%{ }^{6}$. Entre as mulheres que expressaram o desejo de reversão, $40 \%$ encontravam-se na faixa etária entre 18 e 24 anos quando submetidas à laqueadura ${ }^{7}$. O falecimento de filhos, parceiros sem filhos anteriores à união atual, a falta de apoio do parceiro para laqueadura, a sua realização durante ou logo após o parto e a mudança de parceiro após a esterilização cirúrgica são os fatores mais freqüentemente associados com a solicitação ou realização de reversão da laqueadura tubária ${ }^{3,5}$.

Nas últimas três décadas, a reanastomose microcirúrgica foi realizada para a reversão de esterilização tubária naquelas pacientes que manifestaram arrependimento e interesse por novas gestações ${ }^{5}$, tendo sido, por décadas, o procedimento tradicional a reanastomose microcirúrgica laparotômica ${ }^{6}$. A cirurgia também pode ser realizada em pacientes através de minilaparotomias ${ }^{8}$ e videolaparoscopia, com resultados semelhantes ao tradicional, em regime de internação-dia ${ }^{6,9,10}$.

Para bom prognóstico da cirurgia de recanalização tubária, é necessário que os outros parâmetros de fertilidade estejam dentro do limite da normalidade ou que sejam tratáveis ${ }^{11}$. Sabe-se que a fertilidade feminina declina com a idade, sendo a diminuição do número de folículos ovarianos, o principal fator relacionado ${ }^{12}$. Para avaliar a reserva ovariana, o número de folículos antrais e a mensuração sérica basal, no terceiro dia do ciclo menstrual, do hormônio folículo-estimulante (FSH) e do estradiol são freqüentemente utilizados, considerando critério de normalidade $\mathrm{FSH}$ menor que $10 \mathrm{mUI} / \mathrm{mL}$ e estradiol menor que $80 \mathrm{pg} / \mathrm{mL}^{13}$.

O sucesso da reversão é variável e depende de vários fatores como o tipo de esterilização realizada, a idade da mulher quando submetida à laqueadura, o tempo decorrido entre a esterilização e a reversão, a idade e o índice de massa corpórea da mulher no momento da recanalização, o comprimento final da tuba uterina após a anastomose e se a reversão foi possível em uma ou em duas trompas ${ }^{7,14-17}$.

Há grande diferença entre os índices de sucesso alcançados pelos diversos serviços que se propõem a realizar o tratamento, com as taxas de nascidos vivos após a reversão, variando entre 40 a $80 \%$, e a de permeabilidade tubária definida por histerossalpingografia pós-cirúrgica de até $88,8 \% \%^{1,7,8,10,14,18}$. Há relato de risco aumentado de gravidez ectópica após a reversão da laqueadura. O risco desse tipo de gestação na população geral nos Estados Unidos, em 1992, foi de $2 \%{ }^{19}$. Analisando os fatores prognósticos da recanalização tubária, foi constatada uma freqüência de 7,7 a $10 \%$ de gestação ectópica após recanalização ${ }^{15,18}$.

Diante da diversidade dos índices de sucesso apresentados pelos diversos serviços que realizam a cirurgia de reversão da laqueadura tubária, avaliaram-se os resultados alcançados em um serviço público do Distrito Federal. O objetivo deste estudo foi verificar, após 30 anos de experiência, a taxa de gestações intra-uterinas entre as pacientes que foram submetidas à recanalização tubária, o índice de gestações ectópicas após o tratamento e a influência da idade e do tempo decorrido entre a laqueadura e a recanalização (ITLR) sobre o sucesso terapêutico.

\section{Métodos}

Este estudo é de caráter retrospectivo descritivo, efetuado pelo levantamento de informações constantes dos prontuários das pacientes submetidas à recanalização tubária laparotômica, em hospital público do Distrito Federal, de outubro de 1977 a março de 2006.

$\mathrm{O}$ acesso ao registro das pacientes fez-se através do livro de marcação de cirurgia do setor de reprodução humana, única documentação presente e conservada desde a década de 1970. Foram obtidos 138 prontuários do Arquivo Hospitalar Integrado. Não houve contato pessoal com as pacientes, apenas análise de prontuários, após liberação do Comitê de Ética e Pesquisa da Secretaria de Estado de Saúde do Distrito Federal (SES/DF).

De acordo com o protocolo do serviço, as pacientes que se submeteram a recanalização tubária eram encaminhadas do nível de assistência primária do Sistema Único de Saúde (SUS), ao nível terciário por livre demanda, sendo exigidos exame de prevenção do câncer de colo uterino e 
relatório médico. As cirurgias eram realizadas por ordem de chegada ao hospital da paciente ao serviço.

Para verificar o tempo mínimo de seguimento necessário, fez-se o cálculo do tempo médio para primeira gravidez, nos casos avaliados entre os anos de 1977 e 1997 , sendo este período de dez meses. O tempo decorrido entre a cirurgia e a análise dos prontuários variou de 12 meses a 30 anos, sendo estabelecido o período mínimo de um ano de acompanhamento entre a cirurgia e a pesquisa para avaliação do sucesso terapêutico. O período médio de seguimento foi de 14 anos. Por ser um trabalho retrospectivo, realizado em um serviço público, com análise em prontuários de pacientes desde 1977, houve perda de 53 prontuários não localizados e outros 36 não foram empregados devido à ausência de informações relevantes.

Foram excluídos do estudo, os casais que apresentavam também alteração no espermograma do parceiro (seis casos), FSH do terceiro dia do ciclo menstrual acima de $10 \mathrm{mUI} / \mathrm{mL}$ (um caso) e pacientes que apresentavam alterações morfológicas uterinas, tais como pólipos e miomas que comprometessem o endométrio e útero bicorno (11 pacientes) ou que tivessem realizado outra cirurgia tubária como fimbroplastia ou salpingostomia. Pôde-se constatar que, em 13 pacientes, não foi possível realizar a cirurgia devido a alterações tubárias como fimbrectomia bilateral, anexectomia parcial, hidrossalpinge bilateral, cotos tubários curtos e aderências que comprometiam a anatomia e função tubária.

Restaram 71 prontuários para análise final. Destes, foram relatados no estudo como cirurgias de pior prognóstico (CPP), oito pacientes submetidas à recanalização unilateral e sete que necessitaram de extensa adesiólise para que a cirurgia fosse possível.

Utilizou-se a técnica de microcirurgia para anastomose término-terminal dos cotos tubários por laparotomia. Após bloqueio espinhal, realizaram-se incisão suprapúbica, tipo Pfannestiel, e abertura da cavidade abdominal, adotando-se medidas para prevenção de formação de aderências. Verificou-se a possibilidade da realização da cirurgia pela existência de cotos tubários livres que, unidos, medissem quatro ou mais centímetros. Foi realizada a ressecção da parte endurecida dos cotos e a secção de forma, a se observarem a mucosa e a luz tubária. Testou-se a permeabilidade dos cotos através da introdução de fio mononylon 3.0 ou 2.0 por toda a extensão da trompa. Realizou-se a anastomose da muscular tubária com quatro pontos simples, localizados às 12 , nove, seis e três horas, utilizando os fios mononylon 8.0, 7.0 ou 6.0, ou ainda, polydioxanone (PDS) 6.0 ou 7.0. Para sutura de serosa e mesossalpinge foram utilizados os mesmos fios através de ponto contínuo (chuleio simples). Imediatamente antes de a cavidade peritonial ser fechada, deixou-se, aproximadamente, $200 \mathrm{~mL}$ de soro fisiológico heparinizado associado a $10 \mathrm{mg}$ de dexametasona. Suturou-se a parede abdominal por planos. Durante a alta, as pacientes foram orientadas para manter abstinência sexual por 30 dias e retornar após seis meses se não engravidassem, para realização de histerossalpingografia ou, a qualquer momento, em caso de gravidez. Nos casos em que a histerossalpingografia apresentou obstrução tubária, as pacientes foram encaminhadas para fertilização in vitro (FIV) no próprio serviço, desde 1998.

As variáveis coletadas dos prontuários das pacientes foram: a ocorrência de gestação intra-uterina, chegando esta a parto ou a abortamento; a ocorrência de gestação ectópica após a cirurgia; a não-concepção após a reversão; a idade das mulheres na data da recanalização e o tempo decorrido entre a laqueadura e a sua reversão.

O grupo CPP foi analisado à parte, a fim de averiguar possível diferença significativa na freqüência de gestação intra-uterina, em comparação ao grupo de recanalizações bilaterais e livres de aderências, ditas cirurgias de melhor prognóstico (CMP), aplicando-se o teste do $\chi^{2}$.

Nos casos em que se obteve sucesso terapêutico, isto é, gravidez intra-uterina, a idade das mulheres na data da cirurgia de recanalização, e o tempo decorrido entre a laqueadura e a reversão, foi variável, coletadas a fim de correlacioná-las à taxa de gestações como fatores influentes no índice final de gravidez, após a recanalização. As faixas etárias foram subdivididas em grupos entre 20 e 24, 25 e 29,30 e 34, 35 e 39 e entre 40 e 44 anos. Para definição da casuística necessária (n), com $20 \%$ de precisão para cada faixa etária, foi escolhida a prevalência de $60 \%$, que corresponde à taxa de gravidez média do que é observado na literatura pesquisada ${ }^{1,7,10,15,19,20}$. Nas faixas etárias que obtiveram o n necessário para análise estatística, foi verificado se houve diferença significativa $(\mathrm{p}<0,05)$ entre os grupos pelo teste do $\chi^{2}$.

O tempo decorrido entre a laqueadura e a sua reversão variou de um a 18 anos. Para análise da influência do intervalo de tempo de esterilidade sobre o percentual de gravidez, as pacientes foram divididas em três grupos com variação de seis anos: entre um a seis anos, sete a 12 anos e 13 a 18 anos. Nesse quesito não foram contabilizados como gravidez os casos de gestação ectópica, visto que a mesma não pode ser considerada como sucesso terapêutico. Foi aplicado o teste do $\chi^{2}$ de forma a verificar a presença de diferença significativa $(\mathrm{p}<0,05)$ entre os grupos.

\section{Resultados}

Foram realizadas $56 \mathrm{CMP}$, isto é, as reversões bilaterais, sem aderências extensas, resultando em gravidez em 41 casos, sendo 38 tópicas e três ectópicas (Figura 1). Das gestações tópicas, $89,5 \%$ evoluíram até o parto e $10,5 \%$ para abortamento. 


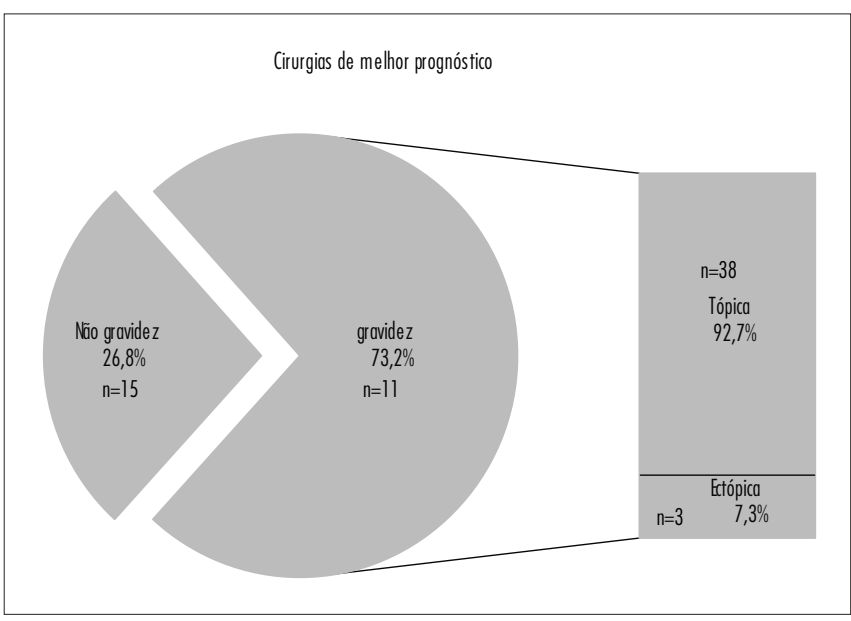

Figura 1 - Resultados obtidos no grupo de cirurgia com melhor prognóstico (52 casos).

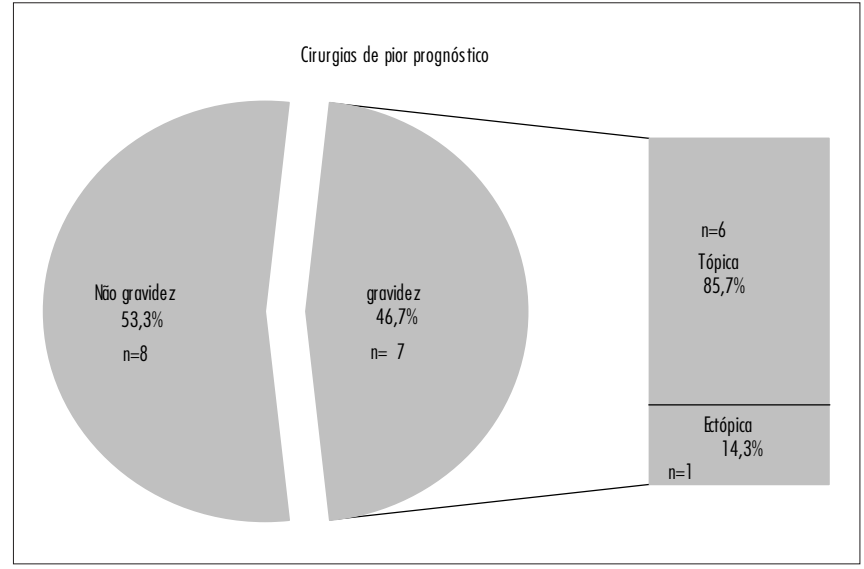

Figura 2 - Resultados obtidos no grupo de cirurgia com pior prognóstico (15 casos). Não gravidez: oito; gravidez: sete (seis tópicas e uma ectópica).

Tabela 1 - Gestações tópicas (em números absolutos e em percentuais) dos casos CMP, CPP e total, por faixas etárias

\begin{tabular}{|c|c|c|c|c|c|c|c|c|c|}
\hline \multirow[t]{3}{*}{ Faixa etária (anos) } & \multicolumn{3}{|c|}{ CMP } & \multicolumn{3}{|c|}{ CPP } & \multicolumn{3}{|c|}{ Total } \\
\hline & \multirow{2}{*}{$\frac{\text { Cirurgias }}{n}$} & \multicolumn{2}{|c|}{ Gravidez tópica } & \multirow{2}{*}{$\frac{\text { Cirurgias }}{n}$} & \multicolumn{2}{|c|}{ Gravidez tópica } & \multirow{2}{*}{$\frac{\text { Cirurgias }}{n}$} & \multicolumn{2}{|c|}{ Gravidez tópica } \\
\hline & & n & $\%$ & & n & $\%$ & & n & $\%$ \\
\hline $20-24$ & 3 & 2 & 66,6 & 0 & 0 & 0 & 3 & 2 & 66,6 \\
\hline $25-29$ & 12 & 7 & 58,3 & 3 & 1 & 33,3 & 15 & 9 & 60 \\
\hline $30-34$ & 21 & 15 & 71,4 & 5 & 3 & 60 & 26 & 18 & 69,2 \\
\hline $35-39$ & 14 & 11 & 78,6 & 6 & 2 & 33,3 & 20 & 13 & 65 \\
\hline Total & 56 & 38 & - & 15 & 6 & - & 71 & 45 & - \\
\hline
\end{tabular}

CMP: casos de melhor prognóstico; CPP: casos de pior prognóstico.

Entre os casos de CPP, isto é, nas quais somente foram possíveis anastomoses unilaterais ou aqueles com extensas aderências, foram realizadas 15 cirurgias, obtendo-se sete gestações, sendo seis tópicas e uma ectópica (Figura 2). Das gestações intra-uterinas, 83,3\% evoluíram até o parto e $16,6 \%$ para abortamento. Neste grupo, oito reversões foram unilaterais e obtiveram $37,5 \%$ de gestação intrauterina. Na revisão dos prontuários foi constatado que, em um dos casos de gravidez ectópica do grupo CMP e no único caso de ectópica no grupo CPP, houve gravidez tópica posterior que evoluiu até o parto.

Associando os resultados encontrados no grupo CMP e CPP, encontrou-se que, de 71 pacientes em 48, ocorreu gravidez, formando um percentual total de $67,6 \%$, sendo $62 \%$ de gestações tópicas e 5,6\% de ectópicas. Não foram encontradas diferenças significativas quando se compararam as taxas de gravidez entre os casos de CMP $(73,2 \%)$ e CPP (46,6\%), sendo $\mathrm{p}=0,10$. O tempo médio de dez meses entre a cirurgia e a primeira gravidez manteve-se constante, não havendo diferença entre os grupos CMP e CPP.

Coletando os dados para analisar a variável idade como fator influente no sucesso terapêutico, isto é, número de gestações tópicas, esta foi dividida em subgrupos (Tabela 1). Foi aplicado o teste do $\chi^{2}$ apenas para as faixas etárias que apresentaram uma casuística maior ou igual a 12 (idade entre 25 e 39 anos), segundo cálculo do n necessário para análise estatística, não sendo encontradas diferenças significativas $(\mathrm{p}=0,8)$, quando se comparou a taxa de gravidez para estas faixas etárias.

Quanto ao intervalo de tempo decorrido entre a ITLR, houve um mínimo de um ano e um máximo de 18 anos.

A ITLR foi dividido em três grupos, com intervalo de seis anos entre eles. No grupo com ITLR de um a seis anos, foram realizadas 22 recanalizações, com $59 \%$ de gravidez. Entre sete e 12 anos, 30 cirurgias, com 66,6\% de gestação e entre 13 e 18 anos, 14 reanastomoses tubárias, com 57\% de gestação. Foram excluídas desta comparação cinco cirurgias, pois nos prontuários não constava a ITLR. Aplicando o teste do $\chi^{2}$, não foram encontradas diferenças significativas quando se compararam as taxas de gravidezes das três ITLR $(\mathrm{p}=0,7)$.

\section{Discussão}

Há três décadas, a recanalização tubária tem sido realizada para reversão de esterilização cirúrgica na mulher que manifesta arrependimento e deseja novas gestações ${ }^{5}$. Há grande número de trabalhos publicados 
sobre o assunto, e as referências bibliográficas, mesmo dos trabalhos atuais, são compostas, além de estudos mais recentes, de literatura que data da década de $1980^{6,7,16,19,20}$. Este hospital público do Distrito Federal tem realizado o procedimento desde 1977.

Dos 56 prontuários analisados em que houve recanalização tubária bilateral ocorreu gestação em 73,2\%. Há discrepâncias na literatura no que se refere ao sucesso terapêutico da reversão bilateral. Encontra-se referência de 45 a $80 \%{ }^{1,10,14,17}$. Em um estudo clássico, com uma amostra de 1.118 pacientes, obteve-se uma taxa de $54,8 \%$ de gravidez $^{17}$. Entre os nossos casos, as recanalizações unilaterais levaram ao índice de gestação tópica de 37,5\%, próximo ao valor de $40 \%$ encontrado na literatura ${ }^{14}$. Do total de 44 pacientes que tiveram gestações tópicas, cinco evoluíram para abortamento, gerando um índice de $11,4 \%$, menor que a taxa de $18 \%$ encontrada por outros autores ${ }^{18,21}$.

Sobre a frequiência de gravidez ectópica, um trabalho em que se analisaram os resultados da recanalização laparoscópica, observou-se um índice de $2,5 \%{ }^{10}$. Outros estudos, no entanto, citam percentual mais elevado, em torno de $8 \%{ }^{15,17}$. Portanto, a taxa de $5,6 \%$ obtida neste serviço é compatível com o exposto na literatura. Comparando os dados encontrados com o risco de gravidez ectópica de $2 \%$ nos Estados Unidos em $1992^{19}$, observa-se um aumento importante do risco após a recanalização.

Foi observado, ainda que não fosse objetivo da pesquisa apresentar seguimento das pacientes após a primeira gestação, que uma das três pacientes no grupo CMP e a única do grupo CPP que apresentou gestação ectópica tiveram uma gestação tópica posteriormente. Fica evidente uma característica do tratamento de esterilidade por laqueadura pela cirurgia de recanalização tubária, em que a paciente, obtendo sucesso na reversão, pode, sem ônus, gestar quantas vezes forem possíveis, se assim desejar, incluindo, no caso de gravidez ectópica, a possibilidade de nova gravidez tópica.

Associando os resultados encontrados no grupo CMP e CPP, obteve-se um percentual de 67,6\% de gravidez. Comparando os percentuais de gravidez do grupo de reversões bilaterais com os encontrados no grupo de reversões unilaterais e/ou adesiólise, pôde-se notar uma diferença de 26,6\% entre eles, porém não é significativa. Deve ser observado que a probabilidade de que os resultados sejam iguais para os dois grupos é de apenas $10 \%(\mathrm{p}=0,10)$, sendo possível especular que, com uma casuística maior, talvez fosse alcançada essa diferença significativa.

As faixas etárias de 20 a 24 e de 40 a 44 anos não alcançaram número de casos suficientes para análise estatística. A faixa etária de menor índice de sucesso alcançado foi entre 20 e 24 anos $(33,3 \%)$. O esperado, sabendo que a fertilidade declina com o avançar da idade ${ }^{12,13}$, era que esta faixa etária alcançasse o percentual mais elevado ou, pelo menos, o mesmo que os demais. Porém, a amostra foi de apenas três cirurgias, o que não permite avaliar a influência desse intervalo de idade.

$\mathrm{Na}$ faixa etária de 40 a 44 anos, o tamanho da amostra também foi um fator limitante, porém, o resultado encontrado de $50 \%$ de gravidez intra-uterina nas recanalizações bilaterais equivale ao que é encontrado na literatura ${ }^{20,21}$. Em um trabalho em que se analisou, especificamente, a recanalização tubária em pacientes com 40 anos ou mais, em uma amostra de 47 mulheres, 56\% engravidaram, sendo que sete evoluíram para abortamento. No entanto, porém, apenas $44 \%$ não obtiveram sucesso na recanalização ${ }^{20}$. Não seria justificável, portanto, a exclusão dessas pacientes dos protocolos de cirurgia sem uma avaliação prévia da sua reserva ovariana.

Outra opção de tratamento para as pacientes que apresentam infertilidade secundária à obstrução bilateral das trompas é a FIV, sendo que a taxa de natalidade por ciclo é de $28 \%$ e a taxa de natalidade, após reversão da laqueadura tubária, excede $55 \%{ }^{11}$. No hospital onde foi realizado este estudo, as pacientes têm a oportunidade de se beneficiar com o tratamento via FIV. Porém, para que este processo seja efetuado, demanda longo tempo de espera, pelo grande número de pacientes que necessitam deste tratamento e por ser hospital público de referência no setor que depende da liberação governamental de medicação e material para sua realização. Torna-se evidente a importância da existência da opção cirúrgica no tratamento das pacientes laqueadas que têm o desejo de gestar. E, a partir deste estudo, haverá um esclarecimento maior para as mesmas, quanto às reais possibilidades de gestação através da recanalização tubária neste hospital.

É interessante comparar os resultados obtidos pela recanalização tubária para faixa etária acima de 40 anos, de até $50 \%$, ao que é encontrado no tratamento de FIV. Mulheres com mais de 40 anos que apresentam esterilidade sem causa aparente têm uma taxa de implantação embrionária de $6,1 \%$ e de parto por ciclo de $5 \%$ ou menos ${ }^{13}$. Um trabalho realizado na Austrália comparou os custos de FIV e de recanalização necessários para se conceber um filho nesta faixa etária, concluindo que, em serviços onde esses tratamentos são custeados pelo governo, a reanastomose tubária deveria ser oferecida por ser altamente custo-efetiva ${ }^{20}$. Cabe avaliar que a recanalização leva ao restabelecimento da capacidade de concepção em cada ciclo ovulatório de forma natural, gerando a oportunidade de obtenção de mais que uma única gravidez por paciente.

Nos grupos de 25 a 29, 30 a 34 e 35 a 39 anos, onde o tamanho da amostra foi satisfatório para análise, não houve diferença significativa entre as faixas etárias. $\mathrm{O}$ mesmo foi encontrado por outro autor que avaliou os mesmos grupos etários ${ }^{16}$. Pode se concluir que, entre 25 e 39 anos, há pouca variação no sucesso da cirurgia, 
com $83 \%$ de probabilidade de equivalência entre as faixas etárias estudadas.

Trabalhos têm estudado o tempo de esterilidade por laqueadura como fator prognóstico para a cirurgia de recanalização tubária, tendo sido encontrado literatura a favor ${ }^{14}$ e contra ${ }^{16,17}$ essa influência nos resultados, evidenciando, talvez, que o intervalo entre a laqueadura e a cirurgia não é um fator independente, e sim, dependente de outros fatores, como a idade. Nesta pesquisa, foi relatado como o intervalo de tempo decorrido entre a ITLR. Não houve diferença significativa entre os grupos, talvez porque a idade média tenha sido menor que 38 anos, sendo pouca a probabilidade, independente da casuística, de que o intervalo de tempo entre a laqueadura e a recanalização influencie no sucesso do tratamento ou que isso possa ser modificado $(\mathrm{p}=0,78)$.

Todos os resultados alcançados foram satisfatórios, frente ao que é observado na literatura, evidenciando, como viável, as chances de gravidez para as pacientes que se submeteram ao procedimento de esterilização cirúrgica e que procuram este serviço público objetivando nova gestação.

\section{Referências}

1. Fernandes AMS, Arruda MS, Palhares MAR, Benetti Junior ND, Moreira CM. Seguimento de mulheres laqueadas arrependidas em serviço público de esterilidade conjugal. Rev Bras Ginecol Obstet. $2001 ; 23(2): 69-73$

2. Yossry $M$, Aboulghar $M, D^{\prime}$ Angelo $A$, Gillett W. In vitro fertilisation versus tubal reanastomosis (sterilisation reversal) for subfertility after tubal sterilisation. Cochrane Database Syst Rev. 2006;(3):CD004144.

3. Machado KM, Ludermir AB, da Costa AM. Changes in family structure and regret following tubal sterilization. Cad. Saúde Pública. 2005;21 (6):1768-77.

4. Sociedade Civil Bem-Estar Familiar no Brasil (BEMFAN). Pesquisa Nacional de Demografia e Saúde (PNDS). Rio de Janeiro: BEMFAN; 1996. p. 52-61.

5. Ribeiro SC, Tormena RA, Bedin AAS, Giribela CRG, Izzo CR, Santos NC, et al. Reanastomose tubária laparoscópica: resultados preliminares. Rev Bras Ginecol Obstet. 2002;24(5):337-41 .

6. Wiegerinck MA, Roukema M, van Kessel PH, Mol BW. Sutureless re-anastomosis by laparoscopy versus microsurgical re-anastomosis by laparotomy for sterilization reversal: a matched cohort stud. Hum Reprod. 2005;20(8):2355-8.

7. Moseman $\mathrm{CP}$, Robinson RD, Bates GW Jr, Propst AM. Identifying women who will request sterilization reversal in a military population. Contraception. 2006;73(5):512-5.

8. Slowey M, Coddington CC. Microsurgical tubal anastomoses performed as an outpatient procedure by minilaparotomy are less expensive and as safe as those performed as an inpatient procedure. Fertil Steril. 1998;69(3):492-5

9. Schollmeyer T, Talab Y, Lehmann-Willenbrock E, Mettler L. Experience of laparoscopic tubal surgery at the department of obstetrics and gynecology, University of Kiel, from 1999 through 2000. JSLS. 2004;8(4):334-8.

10. Cha SH, Lee MH, Kim JH, Lee CN, Yoon TK, Cha KY. Fertility outcome after tubal anastomosis by laparoscopy and laparotomy. J Am Assoc Gynecol Laparosc. 2001;8(3):348-52.
11. Gomel V. Reversal of tubal sterilization versus IVF in the era of assisted reproductive technology: a clinical dilemma. Reprod Biomed Online. 2007; 15(4):403-407.

12. Baird DT, Collins J, Egozcue J, Evers LH, Gianaroli L, Leridon H, et al. Fertility and ageing. Hum Reprod Update. 2005; 11 (3): $261-76$

13. Practice Committee of the American Society for Reproductive Medicine. Aging and infertility in women. Fertil Steril. 2006;86(5 Suppl): S248-52.

14. Hanafi MM. Factors affecting the pregnancy rate after microsurgical reversal of tubal ligation. Fertil Steril. 2003;80(2): 434-40.

15. Gordts S, Campo R, Puttemans P, Gordts S. How important is the method of tubal sterilization in the outcome after tubal anastomosis? Fertil Steril. 2007;88 Suppl 1:S222-S223.

16. Figueredo ED, Evangelista CG, Abdalla CM, Silva BTF. Permeabilidade tubária e gestação após reversão da laqueadura. Rev Bras Promoção Saúde. 2006;19(04):209-15.

17. Kim SH, Shin CJ, Kim JG, Moon SY, Lee JY, Chang YS. Microsurgical reversal of tubal sterilization: a report on 1,118 cases. Fertil Steril. 1997;68(5):865-70

18. Cunha GB, Macedo G, Silva PRG, Florêncio RS, Alencastro JB. Recanalizacäo tubária videolaparoscópica póslaqueadura: resultados iniciais. Rev Bras Ginecol Obstet. 1998;20(2): 105-9.

19. National Center for Health Statistics (NCHS). Advanced report of final mortality statistics, 1992. Mont Vital Stat Rep. 1995;43(6 Suppl): $1-75$.

20. Petrucco OM, Silber SJ, Chamberlain SL, Warnes GM, Davies $M$. Live birth following day surgery reversal of female sterilisation in women older than 40 years: a realistic option in Australia? Med J Aust. 2007;187(5):271-3.

21. Glock JL, Kim AH, Hulka JF, Hunt RB, Trad FS, Brumsted JR. Reproductive outcome after tubal reversal in women 40 years of age or older. Fertil Steril. 1996;65(4):863-5. 